Informe especial

\title{
Salud ambiental: conceptos y actividades
}

\author{
Gonzalo A. Ordóñez
}

RESUMEN La finalidad del trabajo es aportar información y propuestas conceptuales que faciliten la tarea de quienes tienen a su cargo la sistematización institucional de la salud ambiental. Se hace un análisis de la noción de "ambiente" para la cual se sugiere una definición, y se examina el lugar de la salud ambiental en el contexto de los problemas ambientales y sus vertientes "verde" $y$ "azul". Se examinan denominaciones equivalentes de salud ambiental y se introducen los servicios de salud ambiental. Se proporcionan varias definiciones y se da la oficial de salud ambiental adoptada por la OMS en Sofía, Bulgaria (1993). A continuación se transcriben las áreas básicas que a la salud ambiental le han asignado diversas organizaciones o reuniones, como la OPS, la OMS, el Programa 21 y otros. A partir de aquí se construye un repertorio bastante completo de áreas y subáreas y se encuentra que todos los listados son, en realidad, una reunión asistemática de tres tipos de constituyentes: determinantes (factores o hechos de la realidad física), procesos (conjuntos de intervenciones) y funciones (conjuntos de acciones de gestión), los cuales pueden enfocarse matricialmente y llevan a individualizar actividades de los servicios de salud ambiental. Se proponen unas reglas de operación que permiten, en una especie de álgebra, construir expresiones para especificar con precisión las actividades y sus agregados. De este modo se logra disponer de un lenguaje simbólico común que puede ayudar a la intercomunicación, enseñanza e investigación en el ámbito de la salud ambiental.

\section{ANTECEDENTES}

Este artículo se elaboró pensando en los directivos, profesionales, técnicos y demás personas implicadas en el manejo de las complejas relaciones entre el ambiente y la salud humana. La expresión "salud ambiental" o sus equivalentes forman parte del léxico de trabajo de todos ellos y hoy en día suelen aplicarse al respectivo cuerpo de disciplinas científico-técnicas tanto como al cuerpo institucional que las instrumenta y administra. La intención principal del autor fue aportar informa-

\footnotetext{
1 Organización Panamericana de la Salud/ Organización Mundial de la Salud, Ecuador. Toda la correspondencia debe ser enviada a la siguiente dirección postal: Gonzalo Ordóñez, Casilla 09-04247. Guayaquil, Ecuador. Correo electrónico: gaurodec@netscape.net
}

ción, criterios y propuestas conceptuales que pudieran ser de utilidad en la sistematización institucional de la salud ambiental.

\section{TERMINOLOGÍA}

\section{El ambiente}

Las palabras "ambiente", "medio ambiente" y "medio" poseen numerosos sentidos lingüísticos, pero, para nuestros fines concretos de salud ambiental vamos a considerar que son sinónimos y, para simplificar, emplearemos en el texto solamente "ambiente".

Antes de la aparición de los humanos, todo lo que existía en la Tierra (la suma de los ecosistemas del planeta) podía ser llamado "ambiente físico", es decir el conjunto de componentes sólidos, líquidos y gaseosos (litosfera, hidrosfera y atmósfera) con los seres vivos que en ellos se desenvolvían (animales, plantas, hongos, protistos y virus). Una vez que Homo sapiens pasó a formar parte consciente del ambiente físico, pudo considerarlo como la totalidad del mundo real animado e inanimado que lo rodeaba, incluidos los demás seres humanos.

Nótese que, en la práctica actual, cuando se habla de "ambiente" lo usual es que nos estemos refiriendo a "ambiente físico", a menos que se especifique otra cosa, y que la expresión "ambiente natural" suela reservarse para el ambiente físico con exclusión de los humanos y sus obras.

Un sumario de lo anterior puede darse a través de la siguiente definición de "ambiente" que propone el autor: 
Ambiente es, para cada ser o grupo humano, la totalidad del mundo físico que lo rodea, incluidas las entidades vivientes, los demás seres o grupos humanos y sus interrelaciones.

Al estar la gente inmersa en el ambiente surgirán casi inevitablemente una serie de situaciones de interacción que traen consigo la necesidad de manejar y resolver una multitud de "problemas ambientales". En la segunda mitad del siglo XX se hizo muy visible una diferenciación en el enfoque (sobre todo gubernamental) de los problemas ambientales. Por un lado apareció la vertiente "verde", preocupada por los efectos de la actividad humana sobre el ambiente natural y con aspectos como el desarrollo sostenible, la pobreza, la dinámica demográfica, el efecto de invernadero, el deterioro de la capa de ozono, el ordenamiento territorial, la deforestación, la desertización y sequía, las zonas de montaña, la biodiversidad, la biotecnología, la protección de océanos, mares y costas, etc. Por otro lado apareció la vertiente "azul", preocupada por los efectos del ambiente sobre la salud y bienestar de la humanidad. Esta última vertiente es lo que generalmente se ha denominado "salud ambiental". Naturalmente que las dos instancias se hallan íntimamente relacionadas, con temas traslapados o que deben tratarse en ambas, $y$ resulta a veces difícil o artificial establecer una categorización de contenidos en cada una. Pero tal categorización es necesaria, dada la actual división del trabajo en el ámbito tanto nacional como internacional, en la que el Programa de Naciones Unidas para el Medio Ambiente encabeza la vertiente verde y la Organización Mundial de la Salud (OMS) la vertiente azul.

Puesto que hoy en día la denominación "salud ambiental" designa no solo un conjunto analítico de conocimientos y prácticas sino también el sistema de recursos humanos, físicos, financieros e institucionales que trabaja con tales conocimientos y prácticas, es necesario deslindar adecuadamente ambos aspectos. Para ello llamaremos salud ambiental (SA) al conjunto analítico, y servicios de salud ambiental (SSA) al sistema de recursos.

\section{La salud ambiental}

Existen numerosas denominaciones equivalentes de salud ambiental, como "higiene del medio", "saneamiento ambiental", "protección y desarrollo del ambiente", "salud y ambiente", "ambiente y salud" y otras. En mi opinión todas ellas significan básicamente lo mismo, si bien su contenido específico puede diferir mucho entre una $u$ otra entidad o país y responder a circunstancias concretas. La expresión "saneamiento ambiental", que en varios países todavía se utiliza para significar "salud ambiental", comienza a caer en desuso por resultar limitada y confusa. Limitada porque implica una acción curativa, similar a "sanar", cuando una faceta esencial de la salud ambiental es la acción preventiva. Confusa porque, en la práctica presente, la palabra "saneamiento" con frecuencia se reserva solamente para las acciones en el campo del manejo de desechos líquidos y excretas.

La expresión "protección y desarrollo del ambiente" tiene una cabida suficientemente amplia como para incluir a la salud ambiental, pero por su misma amplitud podría englobar también toda la vertiente "verde".

Las expresiones "salud y ambiente" y "ambiente y salud" están sujetas a las connotaciones que se quiera, según la persona o entidad a la que se le pregunte. Para algunos, "salud y ambiente" representa literalmente, por un lado, salud (humana), y por otro, ambiente, en cada caso con el alcance que arbitrariamente se fije. Lo mismo puede suceder con "ambiente y salud". Sin embargo, hay opiniones en el sentido de que "salud y ambiente" significa la influencia del ambiente sobre la salud, pero con énfasis en la salud, mientras que "ambiente y salud" indica un énfasis en el ambiente. Estimo que, frente a esas dos expresiones, debe preferirse la de "salud ambiental", excepto si, en verdad, se quiere aludir por separado al ambiente y a la salud.

\section{Los servicios de salud ambiental}

En el seno de virtualmente cada gobierno existe algún tipo de cuerpo técnico-administrativo, o más de uno, cuyos nombres, posiciones institucionales y estructuras suelen variar mucho de un país a otro, que están encargados de planificar e implementar acciones en el campo de la salud ambiental. En el presente documento aplicaremos a estos cuerpos, y a fin de simplificar la nomenclatura, el apelativo único de "servicios de salud ambiental", como ya se dijo.

De acuerdo con la Oficina Regional de la OMS para Europa (EURO/OMS) (1), los objetivos de los servicios de salud ambiental son:

Básicamente, (. . .) proteger y promover la salud ambiental. Su objetivo primario consiste en asegurar mejores condiciones de vida a fin de promover la salud humana. Pueden identificarse varios objetivos secundarios que contribuyen al todo y consisten en desarrollar y hacer cumplir la legislación, promover la participación e involucración del público, influir en el desarrollo de la responsabilidad del gobierno a todos los niveles, y apoyar la investigación académica y práctica. Esta es la teoría; en la práctica, sin embargo, dicha teoría no siempre se materializa y los servicios de salud ambiental pueden tener o ser usados para servir objetivos diferentes de los definidos arriba. (. . .).

Para los servicios de salud ambiental existe una gama aun más amplia de denominaciones y alcances que para la salud ambiental (véanse algunos ejemplos en el cuadro 1).

\section{DEFINICIONES DE SALUD AMBIENTAL}

El documento "Orientaciones estratégicas y prioridades programáticas, 1991-1994" de la Organización Panamericana de la Salud (OPS) (2) denomina "salud y ambiente" a una de las prioridades básicas y en el texto utiliza indistintamente este nombre o el de "salud ambiental". A manera de definición citamos lo siguiente:

La protección ambiental y la reducción de los efectos nocivos del ambiente en la salud se han convertido en requisitos inseparables de los esfuerzos para construir un proceso efectivo y sostenido de desarrollo económico y social. (. . .) El campo de la salud ambiental, sin embargo, no se 
CUADRO 1. Denominaciones y dependencia de los servicios de salud ambiental en diferentes países

\begin{tabular}{|c|c|c|}
\hline País & $\begin{array}{l}\text { Nombre de los servicios } \\
\text { de salud ambiental }\end{array}$ & Entidad superior \\
\hline Botswana $^{a}$ & Unidad de Salud Ambiental & Ministerio de Salud \\
\hline Brasila $^{a}$ & $\begin{array}{l}\text { Departamento de Políticas y } \\
\text { Evaluación Ambiental }\end{array}$ & $\begin{array}{l}\text { Ministerio del Ambiente, Recursos } \\
\text { Hídricos y Amazonia Legal }\end{array}$ \\
\hline Egipto $^{a}$ & Departamento de Salud Ambiental & Ministerio de Salud y Población \\
\hline Filipinas ${ }^{a}$ & Servicio de Salud Ambiental & Departamento de Salud \\
\hline Indonesia ${ }^{a}$ & Dirección de Salud Ambiental & Ministerio de Salud \\
\hline Argentina $^{b}$ & Departamento de Salud Ambiental & Ministerio de Salud y Acción Social \\
\hline Chile $^{b}$ & División de Salud Ambiental & Ministerio de Salud \\
\hline $\begin{array}{l}\text { Colombiab } \\
\text { (Gobernación } \\
\text { Atlántico) }\end{array}$ & División Medio Ambiente y Salud & $\begin{array}{l}\text { Departamento Administrativo de Salud } \\
\text { del Atlántico }\end{array}$ \\
\hline Costa Ricab & $\begin{array}{l}\text { Dirección de Protección al Ambiente } \\
\text { Humano }\end{array}$ & Ministerio de Salud \\
\hline Méxicob & Dirección General de Salud Ambiental & Secretaría de Salud y Asistencia \\
\hline Panamáb & $\begin{array}{l}\text { Subdirección General de Salud } \\
\text { Ambiental }\end{array}$ & Ministerio de Salud \\
\hline Perúb & Dirección General de Salud Ambiental & Ministerio de Salud \\
\hline Puerto Ricob & $\begin{array}{l}\text { Secretaría Auxiliar para Salud } \\
\text { Ambiental }\end{array}$ & Departamento de Salud \\
\hline Rep. Dominicanab & Dirección General de Salud Ambiental & $\begin{array}{l}\text { Secretaría de Estado de Salud Pública } \\
\text { y Asistencia Social }\end{array}$ \\
\hline
\end{tabular}

a Documentos presentados en la Reunión Interregional sobre Guías para el Fortalecimiento de los Servicios de Salud Ambiental. Hacia una mejor acción gubernamental en los determinantes ambientales de la salud. OMS. Ginebra, mayo de 1998.

${ }^{b}$ Documentos presentados en la Reunión Regional sobre Desarrollo institucional de las unidades de salud ambiental de los ministerios de salud. OPS/OMS. San Juan, Puerto Rico, agosto de 1998

agota en el conocimiento del impacto del ambiente sobre la salud sino que abarca también el diseño, la organización y la ejecución de acciones tendientes a impedir o a revertir los efectos nocivos del ambiente sobre la salud humana.

Las “Orientaciones estratégicas y programáticas, 1995-1998" de la OPS (3) adoptaron el nombre de "protección y desarrollo ambiental" para lo que antes se denominaba "salud ambiental" o "salud y ambiente", pero seguían manteniéndola como una de las orientaciones estratégicas fundamentales. El siguiente párrafo de este documento puede servir como definición general:

En respuesta a los compromisos mundiales de preservar, proteger y restaurar el ambiente para salvaguardar el bienestar de las personas y no permitir que el desarrollo ponga en peligro el futuro, será necesario formular programas nacionales para abordar cuestiones relativas al ambiente en general, al de trabajo y a la vivienda, prestando atención particular a los grupos más necesitados y a los problemas más urgentes.
En una revisión de la práctica europea sobre salud ambiental (1) se menciona que

salud ambiental es un término relativamente reciente, que se ha usado para definir tanto una condición que afecta a la salud humana como una disciplina profesional. Más que un término creado, es un término que ha evolucionado. Inevitablemente, con una historia así, ha adquirido diferentes significados para diferentes personas y diferentes países.

\section{Luego indica que}

el término "salud ambiental" ha sido definido por la OMS como lo que abarca aquellos aspectos de la salud y enfermedad humanas que son determinados por factores ambientales. También se refiere a la teoría y práctica de la evaluación y control de los factores ambientales que pueden afectar la salud.

E informa que en 1993, en una reunión consultiva de la OMS celebrada en Sofía, Bulgaria, se propuso la siguiente definición modificada:
La salud ambiental comprende aquellos aspectos de la salud humana, incluida la calidad de vida, que son determinados por factores ambientales físicos, químicos, biológicos, sociales y psicosociales. También se refiere a la teoría y práctica de evaluación, corrección, control y prevención de los factores ambientales que pueden afectar de forma adversa la salud de la presente y futuras generaciones.

En otra publicación de la OMS (4) se acepta la misma definición emitida en Sofía, que es hasta el momento la única oficialmente adoptada.

\section{ÁREAS BÁSICAS SEGÚN LA PRÁCTICA ACTUAL}

Siempre ha habido opiniones diversas sobre cuál es el contenido de la salud ambiental. Por ejemplo, una clasificación de áreas, sin autor especificado y probablemente de comienzos de los años ochenta, era la siguiente:

- Saneamiento

- Agua potable y alcantarillado urbano

- Agua potable y disposición de excretas en el medio rural

- Aseo urbano

- Mejoramiento de la vivienda

- Protección de los alimentos

- Saneamiento integral de playas

- Artrópodos y roedores

- Control de zoonosis

- Higiene de establecimientos especiales

- Vigilancia y control de calidad del servicio de agua para consumo humano

- Riesgos ambientales

- Salud ocupacional

- Plaguicidas

- Toxicología ambiental y del trabajo

- Radiaciones ionizantes

- Accidentes

- Evaluación de impacto ambiental y salud

- Desastres naturales y tecnológicos

- Contaminación sonora

- Recursos naturales

- Vigilancia de la calidad del aire

- Recursos hídricos

- Suelo, subsuelo, flora, fauna y paisaje 
Como puede notarse, hay una gran diversidad de rubros y es evidente la falta de una adecuada sistematización. La definición de "saneamiento" utilizada es la que estaba en boga en los años setenta y que abarcaba mucho más que en los noventa.

La OPS tuvo para la salud ambiental, hacia finales de la década de los ochenta, la siguiente categorización en grandes áreas:

- Agua potable y saneamiento

- Desechos sólidos

- Riesgos ambientales para la salud

- Salud de los trabajadores

Con motivo de la Conferencia de las Naciones Unidas sobre el Medio Ambiente y el Desarrollo (Río de Janeiro, 1992), la OMS preparó el libro "Nuestro planeta, nuestra salud" (5), cuyos principales capítulos y subcapítulos son:

1. Salud, medio ambiente y desarrollo

2. Desafíos globales para la salud y el medio ambiente

- Aspectos demográficos

- Pobreza

- Utilización de recursos

- Políticas macroeconómicas

3. Alimentación y agricultura

- Producción de alimentos

- Alimentos, dieta y salud

- Contaminación de los alimentos

- Agentes biológicos

- Otros agentes tóxicos en los alimentos

- Riesgos y accidentes laborales

- Sustancias químicas de uso agrícola

- Desarrollo agrícola

- Propiedad o tenencia de la tierra

- Efectos de las actividades agropecuarias sobre la calidad del agua

- Silvicultura

4. Agua

- Agua y producción

- Conservación de la calidad del agua

- Enfermedades infecciosas relacionadas con el agua

- Agua y saneamiento

- Cambios en los patrones de enfermedad como consecuencia del tratamiento del agua

5. Energía

- Utilización de energía y salud
- Efectos sanitarios y ambientales de la producción y utilización de la energía

- Combustibles fósiles y contaminación del aire

- Combustibles fósiles y otros efectos sobre la salud

- Energía hidráulica

- Energía nuclear

- Electricidad

- Accidentes

- Problemas adicionales en países en desarrollo

- Contaminación en espacios cerrados por combustibles fósiles

- Contaminación en espacios cerrados por combustibles de biomasa

6. Industria

- Riesgos para la salud de las actividades industriales

- Exposición laboral

- Riesgos para la salud del público

- Vertidos accidentales

- Sustancias químicas tóxicas y eliminación de residuos peligrosos

- Identificación de los efectos sobre la salud

7. Asentamientos humanos y urbanización

- Vivienda y servicios básicos

- Vivienda y salud

- Servicios básicos

- Asistencia de salud

- Educación

- Recogida y eliminación de basuras

- Accidentes de tráfico

- Ruido

- Exposición en la comunidad

- Exposición ocupacional

- Asentamientos y problemas de salud psicosociales

- Efectos para la salud de la urbanización en países en desarrollo

8. Problemas transfronterizos e internacionales

- Transporte a larga distancia de contaminantes atmosféricos

- Movimiento transfronterizo de productos y residuos peligrosos

- Disminución del ozono estratosférico

- Cambio climático

- Contaminación de los océanos

- Biodiversidad
Aquí hay, naturalmente, un énfasis en lo global y un tratamiento muy integral de cuestiones tanto de la vertiente "verde" como de la "azul".

En 1992, el Programa 21 (6) dedicó 7 de los 14 capítulos de la sección "Los recursos para el desarrollo" al área de la salud ambiental, a pesar de que esta no se menciona explícitamente. Los contenidos son:

- Capítulo 9: Protección de la atmósfera

- Consideración de las incertidumbres: perfeccionamiento de la base científica para la adopción de decisiones.

- Promoción del desarrollo sostenible:

- Desarrollo, eficiencia y consumo de la energía

- Transporte

- Desarrollo industrial

- Desarrollo de los recursos terrestres y marinos y aprovechamiento de las tierras

- Prevención del agotamiento del ozono estratosférico

- Contaminación atmosférica transfronteriza

- Capítulo 10: Enfoque integrado de la planificación y la ordenación de los recursos de tierras

- Capítulo 18: Protección de la calidad y el suministro de los recursos de agua dulce: aplicación de criterios integrados para el aprovechamiento, ordenación y uso de los recursos de agua dulce

- Ordenación y aprovechamiento integrados de los recursos hídricos

- Evaluación de los recursos hídricos

- Protección de los recursos hídricos, la calidad del agua y los ecosistemas acuáticos

- Abastecimiento de agua potable y saneamiento

- El agua y el desarrollo urbano sostenible

- El agua para la producción sostenible de alimentos y el desarrollo rural sostenible

- Repercusiones del cambio climático en los recursos hídricos

- Capítulo 19: Gestión ecológicamente racional de los productos 
químicos tóxicos, incluida la prevención del tráfico internacional ilícito de productos tóxicos y peligrosos

- Expansión y aceleración de la evaluación internacional de los riesgos de los productos químicos

- Armonización de la clasificación y el etiquetado de los productos químicos

- Intercambio de información sobre los productos químicos tóxicos y sobre el riesgo que entrañan los productos químicos

- Organización de programas de reducción de riesgos

- Fomento de la capacidad y los medios nacionales para la gestión de los productos químicos

- Prevención del tráfico internacional ilícito de productos tóxicos y peligrosos

- Fortalecimiento de la cooperación internacional relativa a varias de las áreas de programa

- Capítulo 20: Gestión ecológicamente racional de los desechos peligrosos, incluida la prevención del tráfico internacional ilícito de desechos peligrosos

- Promoción de la prevención y la reducción al mínimo de los desechos peligrosos

- Promoción y fortalecimiento de la capacidad institucional en materia de gestión de desechos peligrosos

- Promoción y fortalecimiento de la cooperación internacional en materia de gestión de los movimientos transfronterizos de desechos peligrosos

- Prevención del tráfico internacional ilícito de desechos peligrosos

- Capítulo 21: Gestión ecológicamente racional de los desechos sólidos y cuestiones relacionadas con las aguas cloacales

- Reducción al mínimo de los desechos

- Aumento al máximo de la reutilización y el reciclado ecológicamente racionales de los desechos

- Promoción de la eliminación y el tratamiento ecológicamente racionales de los desechos

- Ampliación del alcance de los servicios que se ocupan de los desechos

CUADRO 2. Contenidos de la salud ambiental según tres organizaciones internacionales

\begin{tabular}{|c|c|c|}
\hline OPS/OMS & EURO/OMS & USAID/WASH ${ }^{c}$ \\
\hline Agua y saneamiento & Agua y saneamiento & Agua y saneamiento \\
\hline Desechos sólidos & & Desechos sólidos \\
\hline $\begin{array}{l}\text { Control de riesgos } \\
\text { ambientales para la salud }\end{array}$ & Trastornos del medio ambiente en el mundo & \\
\hline Salud de los trabajadores & & Salud ocupacional \\
\hline Higiene de la vivienda & Desarrollo del medio urbano & \\
\hline \multirow[t]{2}{*}{ Calidad del agua } & Calidad del agua & \\
\hline & $\begin{array}{l}\text { Inocuidad de los alimentos } \\
\text { Impacto ambiental }\end{array}$ & Higiene de los alimentos \\
\hline \multirow{2}{*}{ Impacto ambiental } & $\begin{array}{l}\text { Calidad del aire exterior } \\
\text { Calidad del aire interior }\end{array}$ & Contaminación del aire \\
\hline & Productos químicos peligrosos & Materiales peligrosos \\
\hline \multirow[t]{3}{*}{ Residuos de hospitales } & $\begin{array}{l}\text { Desechos peligrosos } \\
\text { Biotecnología }\end{array}$ & \\
\hline & $\begin{array}{l}\text { Socorros de urgencia } \\
\text { Tecnologías menos contaminantes }\end{array}$ & Heridas \\
\hline & & $\begin{array}{l}\text { Aguas de desecho } \\
\text { Enfermedades tropicales }\end{array}$ \\
\hline
\end{tabular}

a OPS/OMS = Organización Panamericana de la Salud/Organización Mundial de la Salud.

${ }^{b}$ EURO/OMS = Oficina Regional de la Organización Mundial de la Salud para Europa.

c USAID/WASH = Programa de Agua y Saneamiento de la Agencia de los Estados Unidos para el Desarrollo Internacional.

- Capítulo 22: Gestión inocua y ecológicamente racional de los desechos radiactivos

Hacia 1993 el autor recopiló la información del cuadro 2, que resume contenidos según tres organizaciones y en el que se procuró que estuvieran en la misma línea rubros afines. Obsérvese que hay una absoluta concordancia en asignar el primer lugar al agua y saneamiento, pero luego las diferencias son muy notables. De hecho, ya no vuelve a haber ningún ítem en que coincidan las tres columnas. EURO/ OMS y la Agencia de Estados Unidos para el Desarrollo Internacional (USAID) incluyen aspectos poco tradicionales, como "socorros de urgencia" y "enfermedades tropicales".

En un documento de la OPS/OMS de 1994 sobre funciones de los técnicos de saneamiento se hace la siguiente lista de las 22 áreas programáticas que, según se dice, "compendian todas las actividades desempeñadas por los técnicos de saneamiento, según información recopilada en los países de la Región" (7):

- Agua potable

- Excretas, aguas negras y aguas de lluvia

- Residuos sólidos
- Higiene de alimentos

- Mejoramiento de la vivienda y los asentamientos humanos

- Control de vectores

- Control de zoonosis

- Saneamiento de establecimientos de servicio público

- Saneamiento de lugares de recreo y turismo

- Saneamiento aplicable a los servicios de transporte marítimo, aéreo, terrestre

- Planificación del saneamiento en el desarrollo urbano y rural

- Salud, higiene y seguridad ocupacional

- Control de sustancias tóxicas

- Radiaciones ionizantes

- Prevención de accidentes

- Impacto ambiental y salud

- Medidas sanitarias en casos excepcionales (desastres naturales y tecnológicos, migraciones, epidemias, etc.)

- Prevención y control de la contaminación del medio ambiente

- Prevención de la contaminación de los recursos hídricos

- Prevención de la contaminación de la flora, fauna y paisaje

- Actividades generales y de gestión

- Promoción de la participación comunitaria y la educación sanitaria y ambiental 
En 1998 la protección y el desarrollo ambiental tienen la siguiente distribución, según la OPS/OMS (8):

- Agua y saneamiento

- Residuos sólidos, salud en la vivienda y entornos saludables

- Incorporación de aspectos de salud en el manejo ambiental

- Identificación y control de riesgos ambientales

- Evaluación de riesgos y promoción de la seguridad química

- Salud de los trabajadores

El documento "Orientaciones estratégicas y programáticas, 1999-2002" de la OPS (9), al igual que el del cuatrienio anterior, tampoco contiene la expresión "salud ambiental" sino que conserva la de "protección y desarrollo ambiental" y menciona lo que sigue en cuanto a cooperación técnica:

- Movilización comunitaria y coordinación intersectorial

- Formar recursos humanos en epidemiología y toxicología ambientales

- Fortalecer las funciones de liderazgo y asesoría de los Ministerios de Salud en el manejo de la salud ambiental

- Desarrollar las capacidades locales para operación y mantenimiento de sistemas y servicios

- Promover programas y proyectos sobre la acción del ambiente en la salud de los niños

- Apoyar acciones de atención primaria ambiental

- Promover la actualización de normas y reglamentos sobre calidad de productos y servicios

- Promover la captación, análisis y utilización de datos e indicadores sobre calidad ambiental

- Cooperar en la mejora de aspectos ligados a la salud de los trabajadores:

- Actualización de la legislación y reglamentación

- Mejoría de la calidad del ambiente ocupacional

- Promoción y prevención

- Atención de salud para los trabajadores

- Protección del trabajador infantil
- Impulsar actividades en agua y saneamiento:

- Apoyar la extensión de la cobertura de los servicios

- Cooperar en la elevación de la calidad bacteriológica del agua para consumo humano

- Difundir tecnologías de bajo costo

- Promover la participación de las comunidades, organizaciones no gubernamentales y sector privado en la expansión de los servicios

- Apoyar estudios sectoriales, la reforma y modernización del sector, la formulación de proyectos prioritarios y la movilización de recursos

- Promover una mejor desinfección del agua

- Contribuir a mejorar el manejo de residuos sólidos municipales:

- Promover el fortalecimiento institucional y la capacidad reguladora y ordenadora

- Desarrollar estudios sectoriales, incluso para residuos de hospitales

- Identificar posibilidades de inversiones financieras

\section{UNA LISTA CASI EXHAUSTIVA}

Cuando los servicios de salud ambiental se enfrentan a procesos de organización o modificación institucional deberán, por supuesto, atender entre otros a un aspecto fundamental: los problemas ambientales que se van a manejar. En algunos países la lista de esos problemas podrá ser relativamente corta a efectos de focalizar recursos en lo más prioritario; en otros convendrá centrarse solamente en los problemas que existan en ese momento y postergar aquello que surja en etapas posteriores del desarrollo, y en muchos se deseará tener una lista muy amplia que no deje olvidado ningún aspecto de la salud ambiental.

Con la idea de proporcionar alguna ayuda en la formulación de la lista de esos problemas se ha intentado hacer una recopilación y sistematización de los componentes de la salud ambiental sobre la base de todo lo visto anterior- mente. Se espera que, además, esto permita a los implicados en la salud ambiental una reflexión más profunda sobre la naturaleza y alcance de su disciplina.

Como un primer paso, y mediante el examen de las fuentes bibliográficas (1-10) y otras ${ }^{2,3}$, se ha elaborado una lista organizada en 18 áreas con 80 subáreas (anexo 1) que incluye la inmensa mayoría de los ítems relacionados con la "salud ambiental".

\section{DETERMINANTES, PROCESOS Y FUNCIONES}

Al analizar la lista del anexo 1, así como virtualmente cualquiera de las demás listas transcritas anteriormente o las listas existentes en los países, se encuentra que en realidad contienen una reunión asistemática de tres tipos de categorías conceptuales a las que se denomina constituyentes y se clasifican así: determinantes de la salud ambiental, como por ejemplo "calidad del agua", "desechos sólidos domésticos" o "contaminación acústica por industrias"; procesos de la salud ambiental, como "tratamiento", "control" o "educación", y funciones, como "estudios de factibilidad" o "promoción". Estos constituyentes se pueden definir como sigue:

- Determinantes de la salud ambiental (DetSA) son los factores o hechos de la realidad física ambiental sobre los que se debe actuar a fin de satisfacer necesidades de los seres humanos relacionadas con la salud ambiental. El cuadro 3 presenta una lista lo más completa posible de 64 DetSA en 20 grupos, ordenada de forma arbitraria. Para identificar un DetSA se proponen los siguientes criterios:

1. Debe ser descrito por un sustantivo o por una frase sustantivada, sin adjetivación.

\footnotetext{
2 Strengthening the role of the Ministries of Health in environmental health: rationale and institutional implications. Borrado 6 de mayo de 1998

3 Environmental Health Services in Europe/Draft Questionnaire for Surveyors. Genève: WHO: sin fecha.
} 
CUADRO 3. Serie de los determinantes de la salud ambiental (DetSA)

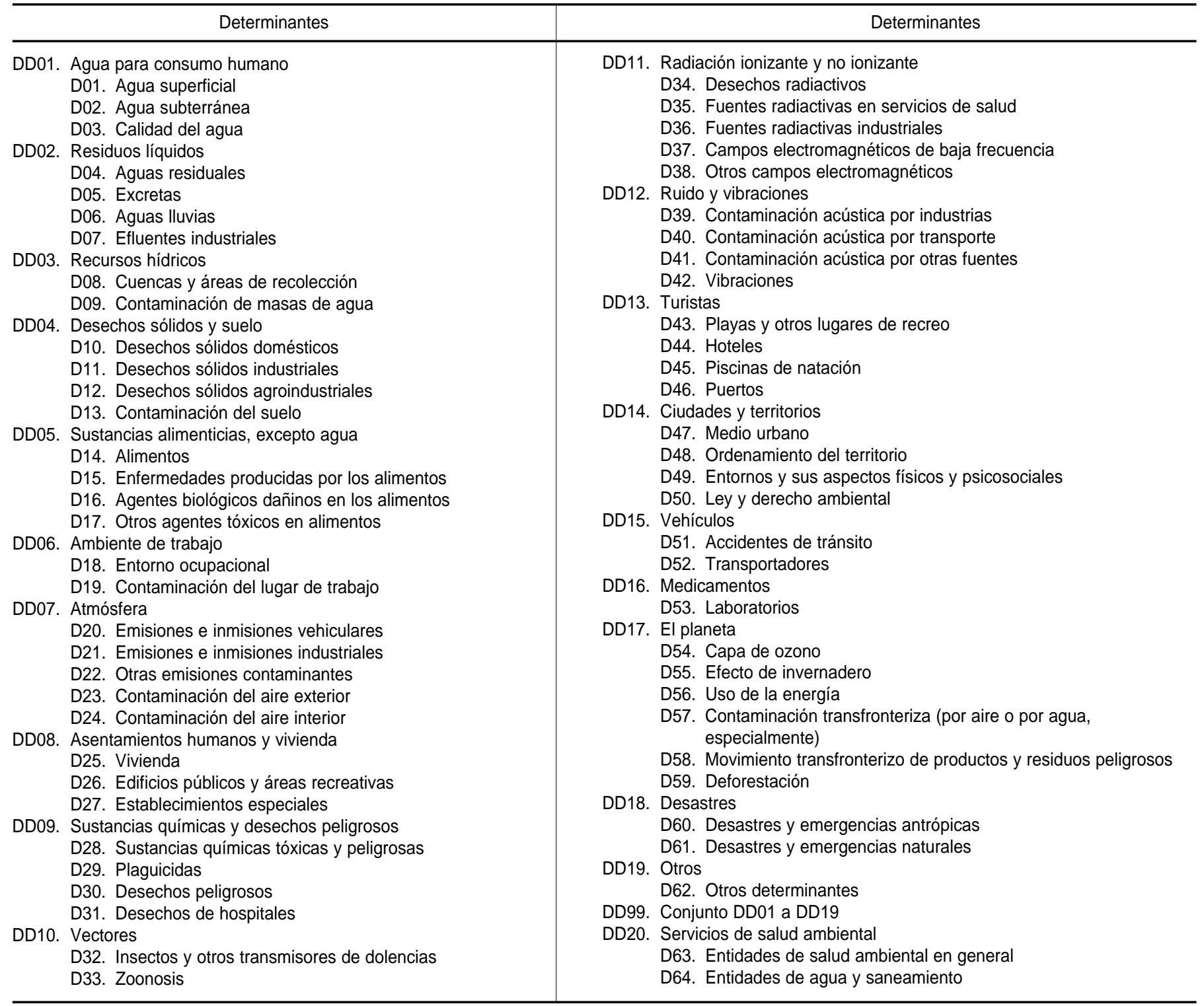

2. Debe ser de nivel razonablemente desagregado. Por ejemplo, en vez de "desechos" habría que decir "desechos sólidos", "aguas residuales", etc.

3. No debe mencionar instalaciones $\mathrm{u}$ otras obras materiales ni intelectuales de salud ambiental, como "relleno sanitario", "planta de tratamiento" o "estudio de factibilidad" (que son Fun y ProSA).

- Procesos de la salud ambiental (ProSA) son los conjuntos de intervenciones que, dentro del marco de las fun- ciones, se aplican a los DetSA a efectos de definir un problema ambiental y una actividad de los servicios de salud ambiental. El cuadro 4 presenta una serie bastante completa de ProSA ordenados operacionalmente.

- Funciones (Fun) son los conjuntos de acciones de gestión que se aplican a los ProSA a efectos de completar una herramienta que luego se hará actuar sobre los DetSA para definir un problema ambiental y una actividad de los servicios de salud ambiental. El cuadro 5 presenta una serie de Fun ordenadas operacionalmente. Las Fun no son específicas de la salud ambiental sino que representan los pasos típicos del desarrollo de un proyecto, desde la previsión hasta el uso, pasando por la materialización y todo ello presidido por una función de dirección.

\section{LA MATRIZ ASSA}

Teóricamente, estos tres tipos de constituyentes pueden verse en una 
CUADRO 4. Serie de los procesos en salud ambiental (ProSA) en orden operacional. Aplicación: ProSA sobre ProSA, o ProSA (de) (sobre) DetSA

\begin{tabular}{|c|c|c|c|}
\hline Símbolo & Proceso & $\begin{array}{l}\text { Algunos procesos o unidades } \\
\text { similares o incluidos }\end{array}$ & Alcance \\
\hline P01 & Producción & Toma, captación, recolección, alcantarillado, bombeo & $\begin{array}{l}\text { D01, D02, D04, D05, D06, D07, D10, D11, D12, D28, D29, } \\
\text { D30, D31, D34 }\end{array}$ \\
\hline P02 & Transporte & Conducción, canalización, emisarios, bombeo & $\begin{array}{l}\text { D01, D02, D04, D05, D06, D07, D10, D11, D12, D28, D29, } \\
\text { D30, D31, D34 }\end{array}$ \\
\hline P03 & Tratamiento & $\begin{array}{l}\text { Potabilización, depuración, desinfección, reducción, reuso, } \\
\text { reciclaje, rechazo, relleno, manipulación, filtración, catali- } \\
\text { zadores, precipitación electrostática, desinfestación, etc. }\end{array}$ & $\begin{array}{l}\text { D01, D02, D04, D05, D06, D07, D09, D10, D11, D12,D13, } \\
\text { D16, D17, D19, D20, D21, D22, D28, D29, D30, D31, } \\
\text { D32, D33, D34, D35, D36, D39, D40, D41, D42 }\end{array}$ \\
\hline P05 & Distribución & Conexiones domiciliarias, bombeo, mercadeo & D01, D02, D06 \\
\hline P06 & Destino final & Eliminación, disposición final, descarga, vertido, drenaje & $\begin{array}{l}\text { D04, D05, D06, D07, D10, D11, D12, D28, D29, D30, D31, } \\
\text { D34, D35, D36 }\end{array}$ \\
\hline P07 & Mejoramiento & $\begin{array}{l}\text { P10 a P17 } \\
\text { Desarrollo, conservación }\end{array}$ & $\begin{array}{l}\text { D03, D08, D09, D13, D14, D19, D23, D24, D25, D39, D40, } \\
\text { D41, D42, D47, D54, D55, D56, D57, D58, D59, D60, } \\
\text { D61, D62, D63, D64 }\end{array}$ \\
\hline P11 & Vigilancia & Inspección, muestreo, análisis; vigilancia epidemiológica & D01 a D64 \\
\hline $\mathrm{P} 12$ & Asesoría & Cooperación nacional e internacional & D01 a D64 \\
\hline P13 & Información & Comunicación social; redes & D01 a D64 \\
\hline P14 & Educación ${ }^{\mathrm{a}}$ & Modificación curricular & D01 a D64 \\
\hline P15 & $\begin{array}{l}\text { Participación } \\
\text { social }\end{array}$ & $\begin{array}{l}\text { Participación comunitaria (incluidas organizaciones no } \\
\text { gubernamentales y sociedad civil) }\end{array}$ & D01 a D64 \\
\hline P16 & Investigación & & D01 a D64 \\
\hline P17 & $\begin{array}{l}\text { Evaluación de } \\
\text { riesgos }\end{array}$ & Valoración ambiental & D01 a D64 \\
\hline P99 & P01 a P17 & & \\
\hline
\end{tabular}

P01 a P09: hardware, inversión relativamente alta.

P10 a P17: software, inversión relativamente baja.

a Formal, no formal, informal.

disposición tridimensional \{Fun, ProSA, DetSA\}, sujetos a reglas especiales de operación en que cada coordenada triple define una actividad de los servicios de salud ambiental. Para simplificar, se ha considerado solamente una disposición bidimensional denominada matriz de actividades de los servicios de salud ambiental, o matriz ASSA. Cada celda de la matriz contiene una actividad a desarrollar por los servicios de salud ambiental, según la intersección de las filas (DetSA) y las columnas (Fun y ProSA).

De este modo, las actividades de los servicios de salud ambiental (ASSA) son cada combinación elemental que nace de una Fun actuando sobre un ProSA y sobre un DetSA. Sirven para manejar un problema ambiental específico y constituyen el motivo de la existencia de los servicios de salud ambiental.
Como toda actividad, cada una de ellas debe disponer de un responsable, un objetivo, un plazo, unos recursos y unas tareas. En la práctica los servicios de salud ambiental trabajarán generalmente con agregados de actividades, que pueden confiarse a unidades $o$ personas específicas.

Nótese que el esquema delineado no contiene a las profesiones relacionadas con la salud ambiental (ingeniería sanitaria, ingeniería ambiental, hidráulica, química, microbiología, epidemiología, toxicología, ergonomía, etc.), que participan en ella como instrumentos para la intervención.

Reglas de operación ASSA. Constituyen un mecanismo formalizador que genera una especie de "álgebra de ASSA", en la que se utilizan símbolos matemáticos para denotar formas de combinar los constituyentes. Obsérvese que cada ítem propuesto en las listas de los cuadros 3, 4 y 5 tiene una identificación formada por la letra D, P o F y un número correlativo. Las reglas son:

- Las ASSA tendrán un nombre proveniente de la intersección de una fila (DetSA) y una columna (ProSA y Fun). Por convención el orden será, de izquierda a derecha: Fun, ProSA y DetSA.

- El hecho de aplicar un constituyente a otro se denotará por el símbolo "mayor que" (>), el cual puede interpretarse, por ejemplo, como "sobre", "de" o "para", según se sugiere en la parte superior de los cuadros 4 y 5 .

- La agrupación de constituyentes se señalará por el símbolo de suma (+). Un conjunto grande de constituyentes del mismo tipo puede indicarse simplemente con la letra " $a$ " interca- 
CUADRO 5. Serie de las funciones (Fun) en orden operacional. Aplicación: Fun para Fun; Fun para ProSA

\begin{tabular}{|c|c|c|c|}
\hline Símbolo & Función & $\begin{array}{l}\text { Funciones o herramientas } \\
\text { similares o relacionadas }\end{array}$ & Alcance $^{a}$ \\
\hline F01 & $\begin{array}{l}\text { Toma de decisiones y establecimiento } \\
\text { de política }\end{array}$ & Adquisición de poder por los niveles locales & $\begin{array}{l}\text { Urbano y rural; enfoque de género; grupos } \\
\text { especiales }^{b}\end{array}$ \\
\hline F02 & Coordinación & & \\
\hline F03 & Elaboración de instrumentos legales & Legislación, normalización & Leyes, normas, reglamentos \\
\hline F04 & Supervisión & & \\
\hline F05 & Planificación & Indicadores & \\
\hline F06 & Programación & & \\
\hline F07 & Estudios en general & Modelos matemáticos; SIG & \\
\hline F08 & Análisis de tecnologías apropiadas & & \\
\hline F09 & Estudios de factibilidad & & \\
\hline F10 & Diseños finales & & \\
\hline $\mathrm{F} 11$ & Financiamiento & $\begin{array}{l}\text { Movilización de recursos, tarifación, } \\
\text { asignación de recursos }\end{array}$ & \\
\hline F12 & Contratación & & \\
\hline F13 & Implementación & $\begin{array}{l}\text { Construcción, montaje, fabricación, } \\
\text { producción }\end{array}$ & \\
\hline $\mathrm{F} 14$ & Puesta en marcha & Puesta en vigor & \\
\hline F15 & Funcionamiento & Operación y mantenimiento & \\
\hline F16 & Administración & $\begin{array}{l}\text { Gerencia, modernización, descentralización, } \\
\text { privatización, sostenibilidad }\end{array}$ & Reclamos, sanciones \\
\hline F17 & Monitorización & Seguimiento & \\
\hline F18 & Evaluación & $\begin{array}{l}\text { Indicadores, auditoría, fiscalización, } \\
\text { contraloría }\end{array}$ & \\
\hline F19 & Desarrollo de recursos humanos & Capacitación & \\
\hline F20 & Promoción & & \\
\hline F21 & Abogacía & & \\
\hline F22 & Concesión de permisos & Registro & \\
\hline F23 & Certificación & Acreditación & \\
\hline F24 & Otras funciones & & \\
\hline F99 & F01 a F24 & & \\
\hline
\end{tabular}

Nota: F01 a F04: Dirección; F05 a F10: Previsión; F11 a F14: Materialización, y F15 a F23: Uso.

a Algunos casos particulares.

' Indígenas, niños y otros.

lada entre los dos extremos del conjunto. Por convención, los DetSA, si son varios, se separarán por comas.

- Por comodidad, la lista completa de funciones se llamará F99 y la lista completa de procesos se denominará P99. En cuanto a los determinantes, cada área (por ejemplo, DD03 para "Recursos hídricos" o DD13 para "Turistas") tiene un nombre que se puede emplear para designar el área completa. La lista total de determinantes (exceptuando DD20) se indicará como DD99.

- Se utilizarán los paréntesis curvos ( ), los paréntesis rectos [ ] y los corchetes \{ \} para significar diversos niveles de agrupación de constituyentes.

- Es lícito aplicar una(s) Fun a otra(s) Fun (por ejemplo, F05 > F19) y un(os)
ProSA a otro(s) ProSA (por ejemplo, P07 > P13).

- Puesto que nos hallamos en el ámbito de la salud ambiental, prácticamente no tendrá sentido una combinación sin DetSA a la derecha; sin embargo, puede haber casos de combinaciones sin Fun o ProSA.

- No todas las combinaciones posibles tienen sentido. En el cuadro 4 se han señalado bajo "Alcance" los determinantes a los que cabe aplicar el respectivo proceso. Puede estimarse que, en total, habría unas 14000 actividades elementales posibles, que conformarían el universo de la salud ambiental.

Ejemplos: (F01 > P01) > D01 correspondería a "toma de decisiones sobre la captación (producción) de agua superficial" y $[\mathrm{F} 03>(\mathrm{P} 10+\mathrm{P} 11)]>\mathrm{D} 03$ a “elaboración de reglamentos para control y vigilancia de calidad del agua".

\section{AGREGADOS}

En la práctica existen numerosas maneras de agrupar actividades para formar el tema de trabajo de una persona o unidad dentro de un servicio de salud ambiental; a estos conjuntos los hemos denominado "agregados" como dijimos arriba. Si bien hay varias formas posibles de traducir o transportar un agregado al "álgebra de ASSA", una expresión en símbolos de dicha álgebra tiene casi una única interpretación en palabras y es por tanto más precisa. El cuadro 6 presenta algunos ejemplos de traducciones tomados de la realidad latinoamericana y con la 
CUADRO 6. Ejemplos de la aplicación del "álgebra de actividades de los servicios de salud ambiental"

Programa de control de calidad del agua potable
Programa de salud ambiental y de control y vigilancia sanitaria
Unidad de control de riesgos en alimentos y bebidas
Programa de saneamiento básico
Programa de efectos del ambiente en la salud
Unidad de saneamiento ambiental $^{\mathrm{a}}$
Unidad de higiene y seguridad laboral

Programa de higienización del ambiente físico inmediatoa Unidad técnica ${ }^{a}$

Unidad de permisos y controles ${ }^{a}$

Dirección Ejecutiva de saneamiento básico ${ }^{\mathrm{a}}$

Unidad de análisis de riesgos ${ }^{\mathrm{a}}$

Atención Primaria Ambiental ${ }^{b}$

Abastecimiento de agua potable ${ }^{b}$

Agua y saneamiento ${ }^{\mathrm{b}}$

Auditoría ambiental ${ }^{b}$

Evaluación de impacto ambiental y salud ${ }^{b}$

Salud ambiental ${ }^{\mathrm{b}}$

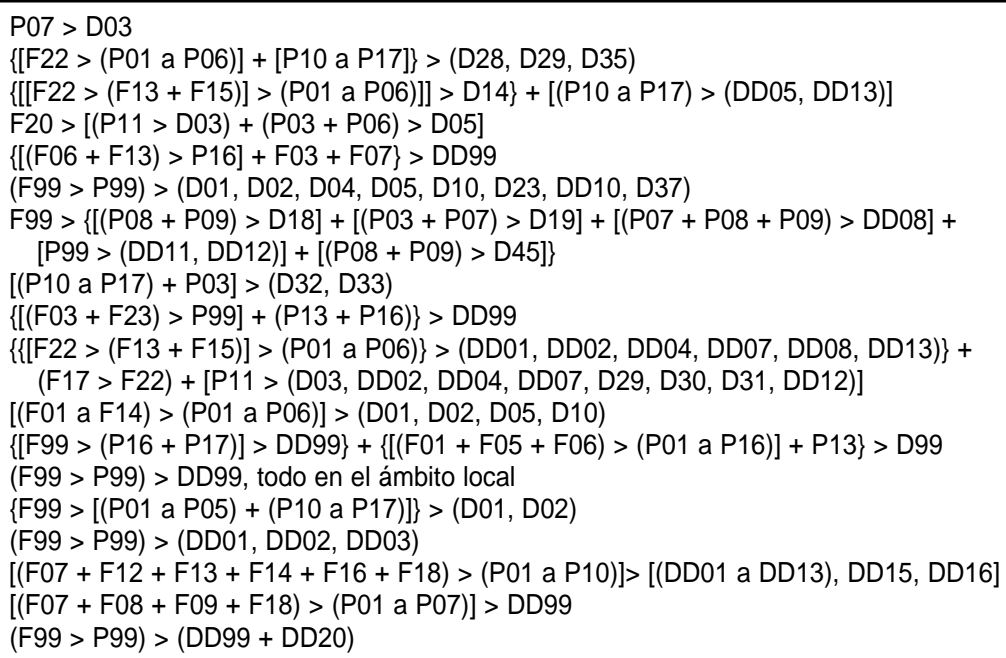

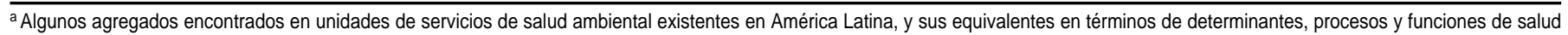
ambiental. Se utilizaron las descripciones dadas por los respectivos servicios de salud ambiental, y el agregado es el nombre de una unidad dentro del servicio de salud ambiental; no se trata necesariamente de ejemplos a seguir. Nótese que a veces no hay una relación clara entre ese nombre y su verdadero ámbito de trabajo.

${ }^{b}$ Ejemplos de posibles equivalencias para otros agregados comunes en la salud ambiental.
}

equivalencia de otros agregados usuales en la salud ambiental.

\section{JUSTIFICACIÓN FINAL}

La matriz ASSA y sus antecedentes creemos que resultan útiles para comprender mejor de dónde surgen y cómo están estructuradas las activida- des de la salud ambiental, al deducirlas como combinaciones sistematizadas de elementos de tres universos específicos (los constituyentes). Con tal herramienta conceptual, además, los encargados de la gestión de los servicios de salud ambiental, y en particular las instituciones del sector público nacional e internacional, dispondrían de una orientación objetiva para fijar y evaluar los alcances de sus acciones y su posición concreta en relación con entidades afines. En este contexto el "álgebra de ASSA" puede ser un medio operativo de intercomunicación, enseñanza e investigación, al revelar la anatomía detallada de los agregados y proporcionar un lenguaje simbólico común más allá de las diferencias idiomáticas y culturales.

\section{REFERENCIAS}

1. MacArthur I, Bonnefoy X. Environmental health services in Europe: an overview of practice in the 1990s. WHO/EURO. Copenhagen, 1997.

2. Organización Panamericana de la Salud. Orientaciones estratégicas y prioridades programáticas, 1991-1994. Washington, DC: OPS 1991. pp. $44-45$

3. Organización Panamericana de la Salud. Orientaciones estratégicas y programáticas, 1995-1998. Washington, DC: OPS; 1995. (Documento oficial 269).

4. Yassi A, Kjellström T, deKok T, Guidotti T. Basic Environmental Health. Ginebra: WHO/ UNEP/UNESCO/CRE; 1998.

5. Organización Panamericana de la Salud/ Organización Mundial de la Salud. Nuestro planeta, nuestra salud. Informe de la Comisión de Salud y Medio Ambiente de la OMS. Washington, DC: OPS/OMS; 1993. (Publicación científica 544)

6. Naciones Unidas. Programa 21. Informe presentado en la Conferencia de las Naciones Unidas sobre el Medio Ambiente y el Desarrollo, Río de Janeiro, junio de 1992.

7. Organización Panamericana de la Salud. Costa Rica. Técnicos en saneamiento. San José: OPS; 1994.

8. División de Salud y Ambiente, OPS. Instructivo de evaluación anual de HEP. Washington, DC, octubre de 1998.

9. OPS/OMS, Comité Ejecutivo, 122a. sesión. Orientaciones estratégicas y programáticas para la Oficina Sanitaria Panamericana
1999-2002. Washington, DC, junio de 1998.

10. Schaefer M. Guidelines for strenghtening environmental health services. Borrador. Presentado en la Reunión Interregional de la OMS sobre Guías para el Fortalecimiento de los Servicios de Salud Ambiental hacia una Mejor Acción Gubernamental en los Determinantes Ambientales de la Salud. Ginebra, mayo de 1998.

Manuscrito recibido el 21 de abril de 1999 y aceptado para publicación, tras revisión, el 13 de octubre de 1999. 
01. Producción y protección de agua segura para bebida

- Fuentes de agua superficial

- Transporte y almacenamiento

- Agua subterránea

- Tratamiento del agua

- Desinfección (incluyendo métodos electrolíticos in situ)

- Distribución del agua

- Calidad del agua (normas, control y vigilancia)

- Agua para industrias

- Gestión y tecnologías

- Políticas de tarifas

02. Alcantarillado y eliminación de excretas

- Recolección y transporte de aguas residuales

- Eliminación in situ de excretas

- Recolección y manejo de aguas lluvias

03. Recursos hídricos y contaminación

- Manejo de cuencas y áreas de recolección

- Hidrogeología

- Contaminación de masas de agua (normas de descarga, control y vigilancia)

- Tratamiento de aguas residuales

- Eliminación de aguas residuales

- Efluentes industriales

- Conservación, reciclaje y reutilización del agua

- Gestión y tarifas

04. Desechos sólidos y protección del suelo

- Normas

- Recolección y transporte de desechos sólidos domésticos

- Tratamiento y eliminación de desechos sólidos domésticos

- Manejo de desechos sólidos industriales

- Reciclaje, reducción, reutilización, rechazo

- Contaminación del suelo
05. Contaminación atmosférica

- Normas

- Monitorización

- Controles de emisión

- Manejo de las emisiones por el transporte

- Contaminación del aire interior

06. Inocuidad de los alimentos

- Normas

- Higiene de los alimentos (producción, distribución, preparación,

venta al menudeo, educación)

- Enfermedades producidas por los alimentos (falta de higiene)

- Uso de plaguicidas

- Control de mataderos

07. Salud y seguridad ocupacional (de los trabajadores)

- Toxicología ocupacional

- Peligros y riesgos ocupacionales

- Contaminantes del lugar de trabajo

- Ergonomía

- Seguridad industrial y agroindustrial

- Normas y medidas correctoras

08. Seguridad química y desechos peligrosos

- Toxicología ambiental

- Manejo de sustancias químicas peligrosas

- Manejo de plaguicidas

- Manejo de desechos peligrosos

- Manejo de desechos de hospitales

- Normas

09. Asentamientos humanos y vivienda

- Normas

- Higiene de la vivienda

- Higiene de edificios públicos y áreas recreativas

- Reparación

- Desarrollo
10. Control de vectores y salud pública veterinaria

- Manejo de vectores en la salud pública

- Artrópodos y roedores

- Control de zoonosis

11. Radiación ionizante y no ionizante

- Normas

- Manejo de desechos radioactivos

- Fuentes radioactivas en servicios de salud

- Fuentes radioactivas industriales

- Campos electromagnéticos y salud

12. Contaminación por ruido

- Normas

- Ruido industrial

- Ruido por transporte

- Ruido por otras fuentes

13. Turismo y salud ambiental

- Playas

- Alojamientos para turistas

- Piscinas de natación

- Controles portuarios de salud

14. Planificación urbana y uso del suelo

- Políticas de desarrollo urbano y rural

- Evaluación de impacto ambiental

15. Seguridad en el transporte

- Prevención de accidentes

16. Calidad de medicamentos

- Bioseguridad de los laboratorios

17. Aspectos ambientales globales

- Disminución de la capa de ozono

- Efecto de invernadero

- Energía y ambiente

- Contaminación transfronteriza

- Gestión ambiental

18. Desastres

- Prevención y mitigación de desastres tecnológicos y naturales

ABSTRACT The objective of this study is to provide information and a conceptual framework that will facilitate the work of persons in charge of systematizing institutions devoted to environmental health. The notion of "environment" is examined and a definition is

Environmental health: concepts and activities proposed, while a look is also taken at the place held by environmental health within the context of environmental problems and their "green" and "blue" components. A number of definitions are put forth before presenting the official definition of environmental health issued by WHO in Sofia (1993). Subsequently a list is presented of the basic areas that have been assigned to the field of environmental health by different organizations or at various meetings, with PAHO, WHO, and Program 21 among them. From this a rather exhaustive list of areas and subareas is constructed, with the finding that all lists are, in reality, an asystematic grouping of three different components: determining factors (from the physical world), processes (types of interventions), and roles (series of administrative tasks) which can be conceived as a matrix and which allow for the description of individual activities by the environmental health services. Certain rules of operation are proposed which make it possible, through a form of algebra, to construct expressions for describing such activities and their aggregates with some precision. Thus, it becomes possible to employ a common symbolic language which can facilitate intercommunication, teaching, and research in the area of environmental health. 